\title{
A Rare Bilaterally Congenital Middle and Distal Tibio-Fibular Synostosis in Northern Malaysia- A Case Report
}

\section{Wong Bor Chern* and Vijaya Kumar L Suppan}

Department of Orthopaedics and Traumatology, Hospital Sultan Abdul Halim, Malaysia

*Corresponding Author: Wong Bor Chern, Department of Orthopaedics and Traumatology, Hospital Sultan Abdul Halim, Malaysia.

Received: July 30, 2019; Published: August 28, 2019

\begin{abstract}
Introduction: Tibiofibular synostosis is an uncommon presentation where a bony bridge develops between the tibia and fibula at any of the mentioned three sites i.e. at the proximal tibiofibular joint, middle tibiofibular joint (in the region of interosseous membrane) or at the distal tibiofibular joint. Distal tibio-fibular synostosis can be congenital or it may be secondary to trauma, surgery, metabolic or genetic condition. Reports of congenital distal tibiofibular synostosis are rare in published literature. We present a case of tibiofibular synostosis that involves the bilateral middle and distal part of the tibia and fibula based on characteristic X-ray findings.

Case Presentation: A 17 years old Malay female patient, currently as a student was referred to district hospital, presented with a history of left anterior shin pain for the past 3 years. Pain was diffuse and dull-aching type, worsening upon prolonged standing and walking long distances. There were no paraesthesiae in the calf or foot. There was no history of trauma or infection. Patient was having limping gait since then however no deformity seen over the left lower limb. Patient visited to clinic for multiple times and was not investigated. She was treated conservatively with analgesics however pain persisted. Symptoms worsen since 6 months ago with similar pain over the right leg and thus was referred to hospital. X-rays over bilateral lower limbs was done which showed multiple bony bridge across tibia-fibula at middle and distal one third of right tibia and at the junction of middle tibia-fibula over the left lower limb. On lateral view the medullary canals appeared to be communicating. Other areas of skeletal survey-revealed no other bony anomalies. Based on conventional imaging a diagnosis of congenital tibiofibular synostosis was given. Clinical examination showed tenderness over the medial side of right tibia and ankle, however range of motion over the right ankle was full. Patient not keen for surgical intervention and was treated conservatively by activity modification and analgesics.

Discussions: Tibio-fibular synostosis is rarely described in orthopedic literature and has diversified causes. There is an ongoing debate on the treatment of choice. Henry., et al. advocate successful treatment by conservative measures while a few others such as Hakan and Flandry support surgical resection.
\end{abstract}

Keywords: Synostosis; Tibiofibular; Bony; Lower Limbs; Periosteal

\section{Introduction}

Tibiofibular synostosis is an uncommon presentation where a bony bridge develops between the tibia and fibula at any of the mentioned three sites i.e. at the proximal tibiofibular joint, middle tibiofibular joint (in the region of interosseous membrane) or at the distal tibiofibular joint. Distal tibio-fibular synostosis can be congenital or it may be secondary to trauma, surgery, metabolic or genetic condition [2]. Reports of congenital distal tibiofibular synostosis are rare in published literature. We present a case of tibiofibular synostosis that involves the bilateral middle and distal part of the tibia and fibula based on characteristic X-ray findings. 


\section{Case Presentation}

A 17 years old Malay female patient, currently as a student was referred to district hospital, presented with a history of left anterior shin pain for the past 3 years. Pain was diffuse and dullaching type, worsening upon prolonged standing and walking long distances. There were no paraesthesiae in the calf or foot. There was no history of trauma or infection. Patient was having limping gait since then however no deformity seen over the left lower limb. Patient visited to clinic for multiple times and was not investigated. She was treated conservatively with analgesics however pain persisted. Symptoms worsen since 6 months ago with similar pain over the right leg and thus was referred to hospital. X-rays over bilateral lower limbs was done which showed multiple bony bridge across tibia-fibula at middle and distal one third of right tibia and at the junction of middle tibia-fibula over the left lower limb. On lateral view the medullary canals appeared to be communicating. Other areas of skeletal survey-revealed no other bony anomalies. Based on conventional imaging a diagnosis of congenital tibiofibular synostosis was given. Clinical examination showed tenderness over the medial side of right tibia and ankle, however range of motion over the right ankle was full. Patient not keen for surgical intervention and was treated conservatively by activity modification and analgesics.

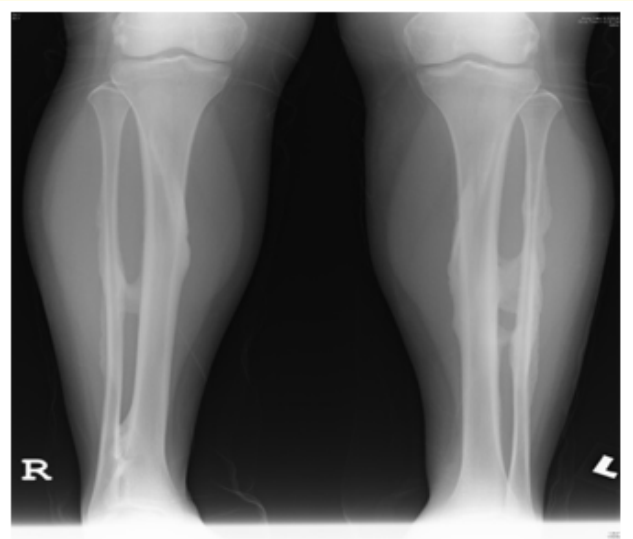

Figure 1: X-rays of bilateral tibia/fibilla (AP View).

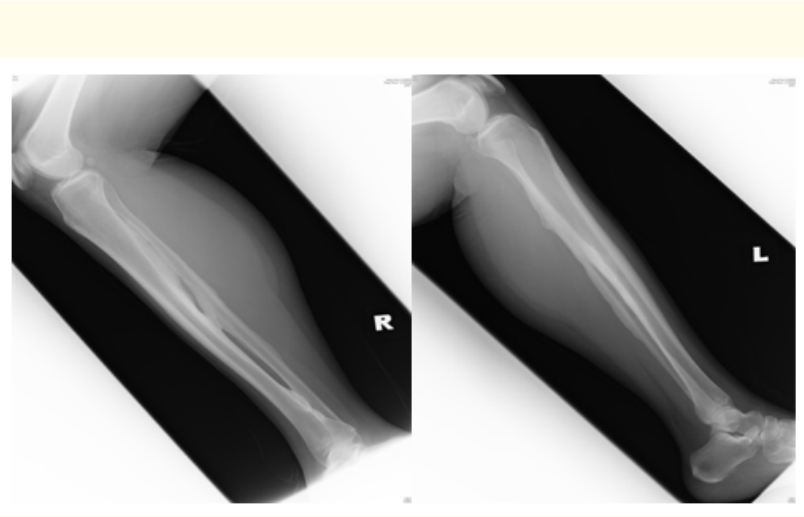

Figure 2: X-rays of bilateral tibia/fibilla (Lateral View).

\section{Discussion}

Tibio-fibular synostosis is rarely described in orthopedic literature and has diversified causes. Proximal synostosis is usually congenital, while the middle and distal joint involvement is mostly acquired [1]. Trauma is the most common cause of distal tibiofibular synostosis [1]. This is a case of bilaterally synostosis of middle and distal tibiofibular joint with no previous history of trauma or injury. Although the middle tibiofibular synostosis is mostly acquired the bilateral symmetry of synostosis with no evidence of callus formation or trauma in the present case favours congenital etiology. It may be secondary to iatrogenic causes or underlying conditions like kissing osteochondroma, fibrodysplasia ossificans progressive (FOP), subperiosteal haemorrhage (as seen in scurvy and hemophilia), and flourosis [1].There are very few isolated case reports of congenital distal tibiofibular synostosis. Synostosis of the middle third of the diaphysis of the tibia and fibula has been described previously in football and basketball players [3,4]. Despite its localization, synostosis appearing as a congenital lesion or occuring before the closure of the physeal plates is usually complicated by shortening of the lateral malleolus, ankle valgus and prominence of the fibular head at the knee. These lesions may potentially lead to ankle pain, since the synostosis interferes with the normal motion that occurs between the tibia and fibula during weight-bearing [6].

Citation: Wong Bor Chern and Vijaya Kumar L Suppan. "A Rare Bilaterally Congenital Middle and Distal Tibio-Fibular Synostosis in Northern Malaysia- A Case Report". Acta Scientific Orthopaedics 2.9 (2019): 61-63. 


\section{Conclusion}

Management in such cases may be conservative or surgical. Treatment should always start with conservative measures, such as rest, ice compression, non-steroidal anti-inflammatory drugs and ultrasound-guided steroid injection. Surgery should be reserved for patients not improving after conservative treatment. Surgical excision should be reserved for the athletically active patient whose symptoms are gradually progressing and disabling, and only after the synostosis appears as mature cortical bone on radiographs.

There is an ongoing debate on the treatment of choice. Henry., et al. [4] advocate successful treatment by conservative measures while a few others such as Hakan and Flandry [5] support surgical resection. In this case, the patient is not an active athlete, thus conservative measures is adequate for her current condition.

\section{Bibliography}

1. O’Dwyer KJ. "Proximal tibio-fibular synostosis. A rare congenital anomaly". Acta Orthopaedica Belgica 57 (1991): 204.

2. Bessler W., et al. "Kissing osteochondromata leading to synostoses”. European Radiology 7.4 (1997): 480-485.

3. James SH., et al. "Tibiofibular synostosis in a professional football player". The Journal of Bone and Joint Surgery 89-B (2007): 109-111.

4. Henry JH., et al. "Tibiofibular synostosis in professional basketball players". American Journal of Sports Medicine 21 (1993): 519-622.

5. Flandry F and Sanders R. "A tibiofibular synostosis an unusual case of shin-splint-like pain". American Journal of Sports Medicine (1987): 280-284.

6. Frick SL., et al. "Altered fibular growth patterns after tibiofibular synostosis in children". Journal of Bone and Joint Surgery 83 (2001): 247-254.

Volume 2 Issue 9 September 2019

C) All rights are reserved by Wong Bor Chern and Vijaya Kumar L Suppan. 